PASQUET, P., OBERTI, B. EL ATI, J. \& HLADIK, C.M., (2002) - Relationships between thresholdbased PROP sensitivity and food preferences of Tunisians. Appetite, 39 : 167-173.

\title{
Relationships between threshold-based PROP sensitivity and food preferences of Tunisians
}

\author{
P. PASQUET*, B. OBERTI*, J. EL ATI** and C.M. HLADIK*** \\ *Centre National de la Recherche Scientifique (France) \\ ** Institut de Nutrition, Tunis (Tunisia) \\ *** Muséum National d'Histoire Naturelle, Paris.
}

\begin{abstract}
The extent to which taste responses - and notably the genetically determined sensitivity to 6-npropylthiouracil (PROP) - influences food preferences and food use is still a matter of debate. We addressed the issue on the basis of a behavioural and anthropological study performed in Tunis in 1999. The working sample consists of 123 adults of both sexes ( 38 men, 85 women), aged 19 to 59 , in various social categories. Taste recognition thresholds for sucrose, fructose, sodium chloride, quinine hydrochloride, citric acid, tannic acid, oak tannin and PROP were determined by presenting, in a semi-randomised order (blind-test), series of graded aqueous solutions of each product. Subjects also tasted and rated the pleasantness/unpleasantness of 4 supra-threshold solutions of $\mathrm{NaCl}$ and sucrose. All subjects completed a checklist of 43 food items representative of Tunisian diet, rated in terms of flavour, cost, effect on health and prestige on a Labelled Affective Magnitude (LAM) scale. According to the underlying distribution of PROP thresholds, the subjects were separated into three categories: "non-tasters", "medium-threshold tasters", and "low-threshold tasters". Results bring out the specificity of low-threshold tasters, as exhibiting a greater taste sensitivity for most tested substances. Low-threshold taster status is also linked to higher mean food preferences ratings irrespective of sex, age and socio-cultural influences. Tasters as a group (medium-threshold tasters + low-threshold tasters) do not exhibit a higher percentage of food dislikes; however PROP sensitivity is negatively correlated with hedonic responses to $\mathrm{NaCl}$ solutions. These results together with the evidence of a limited set of food actually used by low-threshold tasters suggest that these subjects might have difficulties at overcoming an inherent neophobia.
\end{abstract}

\section{INTRODUCTION}

Taste responses have been shaped, as a result of selective pressure, according to nutrient content and toxicity of potential foods. In this context, human taste sensitivity does not functionally differ from that of other primates (Hladik \& Pasquet, 1999; Hladik et al., 2002). Even for hedonics, non-human primates and humans display, in neonates, equivalent innate stereotyped gusto-facial responses with sugars being always accepted, whereas bitter tasting substances are rejected (Steiner et al., 2001). However, human taste preferences and aversions may vary to a large extent during growth and adulthood, as a result of congenital factors, individual experience, and sociocultural influences (Birch, et al., 1980; Rozin, 1990). Studies of feeding behaviour genetics yielded significant results only for taste perception of some bitter substances such as phenylthiocarbamide (PTC), chemical analogs with the $\mathrm{N}-\mathrm{C}=\mathrm{S}$ group (thiourea, 6- $n$-propylthiouracyl or PROP) and quinine (see reviews in: Pasquet, 1994; Reed et al., 1997).

The relationship between genetically-controlled taste sensitivity and food preferences was explored by Fisher et al., (1961), who found a positive correlation between bitter sensitivity to PROP, or to quinine, and the percentage of food dislikes in a list of 118 items. On this ground, later investigations were aimed at using PTC or PROP sensitivity as a genetic marker for feeding behaviour in a perspective of dietary prevention of chronic diseases (review in: Drewnowski, 2000). The taste threshold distribution of PTC or PROP has been classically described as a bimodal distribution allowing the recognition, in the study sample, of "tasters" versus "non-tasters" (Fox, 1932), following a simple dominant/recessive genetic model (Olson et al., 1989). Later, Bartoshuk (1993) distinguished among tasters, a group of "super-tasters" on the basis of bitterness intensity perceived at a supra-threshold level 
(using the ratio PROP response / $\mathrm{NaCl}$ response). This group would correspond to homozygote tasters with peculiar tongue anatomical features (Reddy et al., 1993).

Such findings stimulated recent studies, especially on sensitivity for PROP and food preferences, including the use of cruciferous vegetable containing the $\mathrm{N}-\mathrm{C}=\mathrm{S}$ chemical group, or other phytonutrients (Niewind et al., 1988 ; Smagghe \& Louis-Sylvestre, 1998; Jersza-Latta et al., 1990; Drewnowski, 2000); however, in the attempt at correlating these parameters, no clear-cut, and even paradoxical results, were obtained from these studies. Discrepancies between studies could result from procedural differences and/or distinct socio-cultural backgrounds in the tested groups.

An investigation of PROP threshold distribution of 1015 subjects (Reed et al., 1995) suggested that a trimodal distribution is, at least, as likely as the classical taster/non-taster model, thus revealing the very existence of three underlying groups. Analysing differences in feeding responses between such taste threshold-based categories may help clarify the issue of relationships between bitter sensitivity and feeding behaviour.

Accordingly, we conducted a study exclusively focused on the PROP thresholds to determine the extent to which sensory responses correlate to food preferences, acceptance and use, in a sample of subjects sharing a homogeneous cultural background.

\section{Methods}

This study was carried out at the Nutrition Institute of Tunis (Tunisia), from March to May 1999. Voluntary subjects were 123 inhabitants of Tunis of both sexes (38 men, 85 women), aged 19 to 59 years (mean: 36.0, SD: 10.7), from a panel of previous national nutritional survey. Pregnant women, subjects with pathologies that modify the ability to taste (otitis, rhinitis...), and heavy smokers were excluded from the study. After the participants filled a consent form and a socio-demographic questionnaire, body weight and height were measured. Testing was carried out between 8:00 and 10:30 am, after an overnight fast. After determination of taste thresholds and supra-threshold responses, a standardised light breakfast was provided (between 9:00 and 9:30), before completing checklists and questionnaires.

\section{Determination of taste thresholds}

The staircase-method (modified from Cornsweet, 1962), was used to determine taste recognition thresholds. After informing the subject on the taste categories he or she could be faced with (water, salty, sour, sweet, bitter and astringent), recognition thresholds were measured during a blind test. Nine solutions of sucrose (1.5-400 $\mathrm{mM})$, 10 of fructose $(2.0-1000 \mathrm{mM}), 8$ of sodium chloride $(1.9-250 \mathrm{mM}), 11$ of quinine hydrochloride $(0.4-400 \mu \mathrm{M})$, 7 of citric acid (0.4-25 mM), 12 of tannic acid $(4-8000 \mu \mathrm{M})$, and 9 of oak tannin $(0.02-6 \mathrm{~g} / \mathrm{l})$ were presented in a semi-randomised order, starting with the weakest solution in order of increasing concentration (twofold steps). Sensitivity to 6- $n$-propylthiouracyl (PROP) was investigated after testing the previous substance solutions and a range of 12 PROP solutions $\left(1.910^{-6}-3.810^{-3} \mathrm{M}\right)$ was used. Each solution was spat out and followed by mouth rising, using local drinking water, the same as used for diluting solutions, which salt content is about 20 times lower than that of our below-threshold most diluted salt solution. Once the taste of two successive concentrations was recognised successfully (among the six possible taste categories), an up and down procedure was used until unambiguous recognition was noted. The actual recognition threshold was estimated by the geometric mean of the observed concentration and the next lower one.

\section{Supra-threshold hedonics}

Four solutions of sodium chloride $(125,250,500$ and $1000 \mathrm{mM})$ and four sucrose solutions $(200,400,800$ and $1600 \mathrm{mM}$ ) were provided in ascending order, with two water rinses (with one minute time elapse) between each trial. The perceived pleasantness/unpleasantness of each solution was marked by the subject on a visual analogue $100 \mathrm{~mm}$ vertical scale anchored at each end and midway, labelled with 'maximum pleasantness' on the top, 'neutral' at midway, and 'maximum unpleasantness' at the bottom. The responses were translated into numeric values (positive for pleasant and negative for unpleasant) by measuring on the scale the distance (in mm) between the mark and the neutral point. 


\section{Food preferences and attitudes}

Subjects completed questionnaires on a booklet concerning 43 food items representative of Tunisian diet (Hubert, 1984), presented on separated sheets in random order. Each sheet includes three 11-point labelled vertical scales presented side by side and concerning respectively preference (adapted from the Labelled Affective Magnitude scale; Schutz \& Cardello, 2001), perceived food effect on health, and cost. A labelled box was inserted above each scale to be filled respectively when the food item had never been tried or was unknown, when the subject had no idea on its effect on health, and when he/she had no idea of its cost. Otherwise, responses were noted with a bar on each scale. The results were measured positively or negatively from midway, to be rescaled from -100 to +100 between the two extreme semantic labels. Food items rated below -20 were considered as unpleasant. Extra yes/no answer questions concerning ritual (festive use) and prestige (used only with guests) were added to these questionnaires.

\section{Statistics}

The SKUMIX procedure (McLean et al., 1976) was used to determine the underlying PROP threshold distribution, and STATISTICA version 5.0, for descriptive statistics, group comparison (ANOVA), correlations, principal component analysis, multiple regression analysis, and hierarchical cluster analysis (average linkage method, using the Pearson correlation coefficients to determine the aggregation distances).

\section{Results}

\section{PROP tasting status}

No significant difference between male and female mean taste threshold was found $(\mathrm{t}=0.75 ; \mathrm{p}=0.45)$. Thus, data concerning both sexes were grouped to apply the SKUMIX procedure. SKUMIX yields a distribution of PROP thresholds which is more likely bimodal, or tri-modal (with similar likelihood) than mono-modal $\left(\mathrm{Chi}^{2}=21.2\right.$, $\mathrm{p}<0.001$ ), assuming that variances associated with the distributions are equal.

Figure 1 shows the tri-modal model, which, according to Reed et al., (1996) is the most likely underlying PROP threshold distribution in a large population sample. The anti-modes of distributions are in the range of those

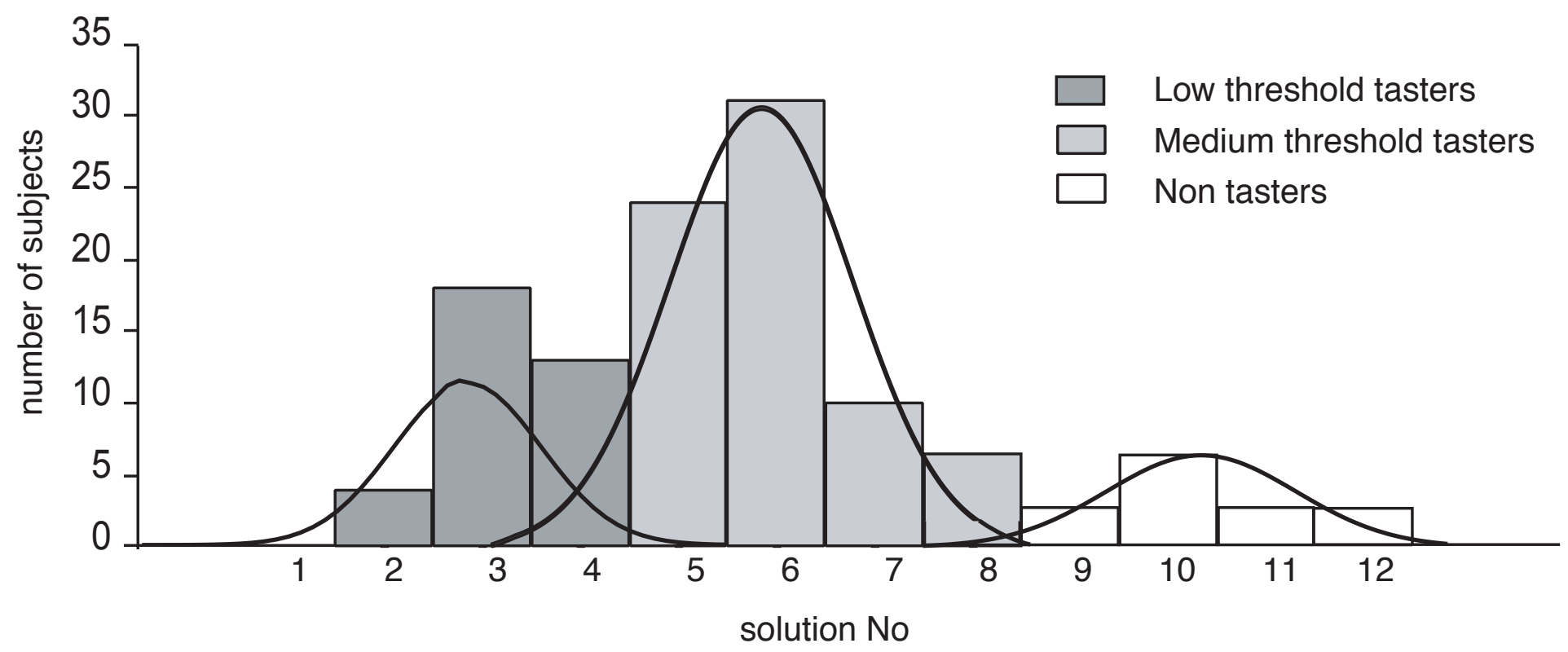

FIGURE 1 - Distribution of PROP taste recognition thresholds, illustrating the three groups defined by the three-distribution model. 
observed in Reed and co-authors' analysis. Accordingly, we classified as "non-tasters" (NT) all subjects with an actual estimated threshold above $1.68 \times 10^{-4} \mathrm{M}$ (equivalent to observed threshold at solution \# 8), and "low threshold taster" (LTT) subjects with a threshold below $2.1 \times 10^{-5} \mathrm{M}$ (equivalent to observed threshold at solution \# 5; our cutoff point being, with the tri-modal model, close to the anti-mode found by Reed and co-authors). Subjects with intermediary recognition threshold are classified as "medium threshold tasters" (MTT). The observed percentages are $28.5 \%$ LTT, $59.3 \%$ MTT, and $12.2 \%$ NT. We did not find any difference in the proportion of subjects of both sexes in the different PROP taster categories $\left(\mathrm{Chi}^{2}=1.34, \mathrm{p}=0.51\right)$.

PROP status and sensitivity to other substances

In Figure 2, we show the results of a comparison of the thresholds of all other tested substances between the LTT group and the rest of the sample (MTT + NT). A lower threshold is generally found in the LTT group, with a significant difference $(\mathrm{p}<0.05)$ for sucrose and fructose. For quinine and tannins, the difference is nearly significant $(\mathrm{p}<0.07)$. Conversely, when comparing the NT group with all tasters (LTT + MTT), no significant difference was found.

However, considering the entire distribution of thresholds (log), PROP sensitivity is weakly correlated with sucrose $(r=0.24, p=0.005)$, quinine $(r=0.21 ; \mathrm{p}=0.02)$ and tannic acid $(\mathrm{r}=0.18 ; \mathrm{p}=0 ; 04)$. Furthermore, as shown in Figure 3, PROP taste sensitivity does not cluster (or very poorly) with the other substances, including quinine and tannins.

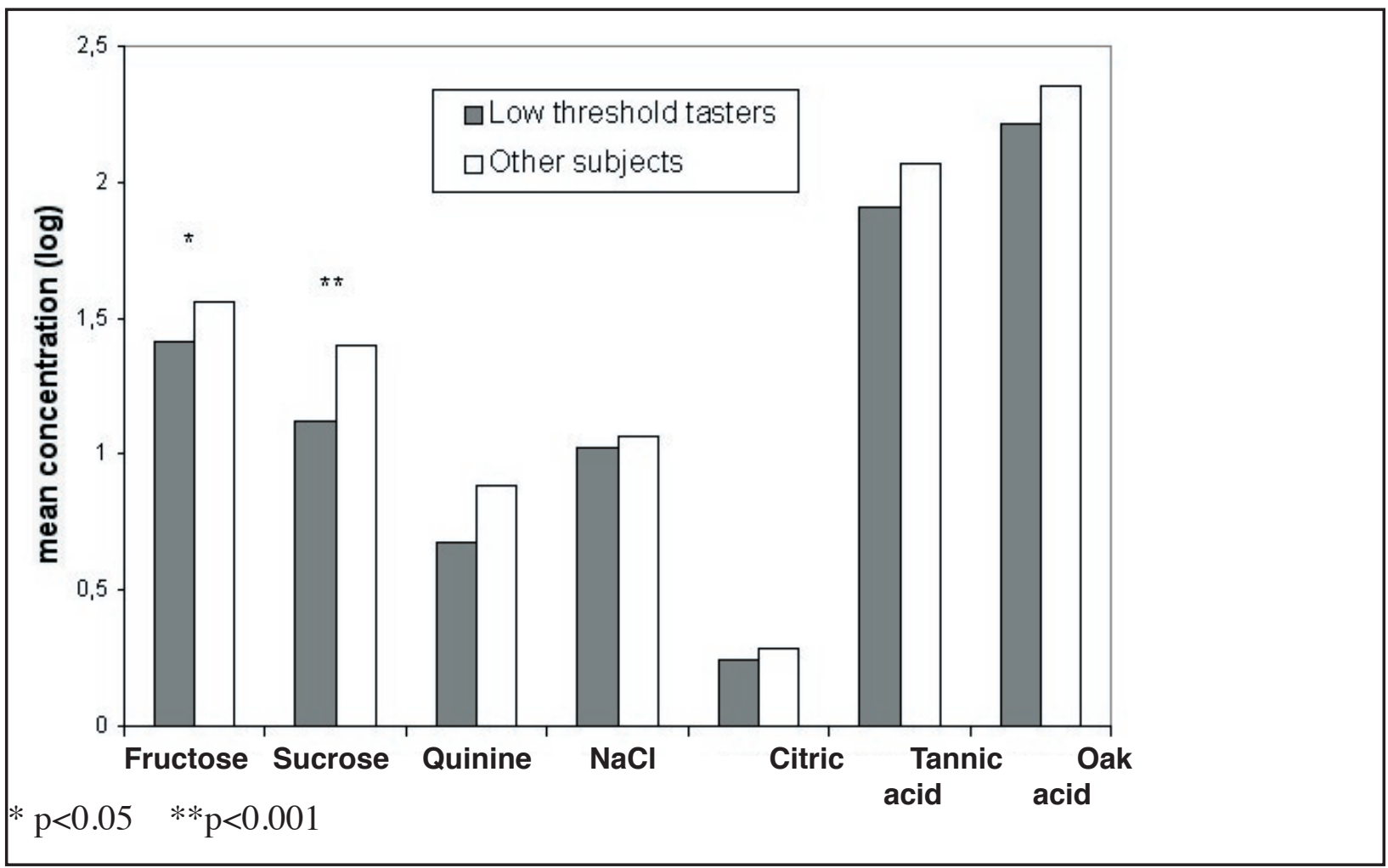

FIGURE $2-$ Mean taste recognition thresholds for various substances among the PROP low threshold tasters (LTT) vs other subjects (MTT $+\mathrm{NT})$.

\section{PROP status and hedonic ratings}

Figure 4 shows a clear inverse relationship between sensitivity to PROP and the mean level of hedonic rating of sodium chloride solutions $[F(8,234)=2.93 ; p=0.004]$. In contrast, no relationship between PROP status and hedonic responses to sucrose was found $[\mathrm{F}(8,234)=0.60 ; \mathrm{p}=0.77]$; likewise, classifying the subjects into 'sweet likers' $(\mathrm{N}=30)$ and 'sweet dislikers' $(\mathrm{N}=70)$, following Drewnowski et al. (1997a), did not reveal any association with the PROP status $\left(\mathrm{Chi}^{2}=3.4, \mathrm{p}=0.2\right)$. 


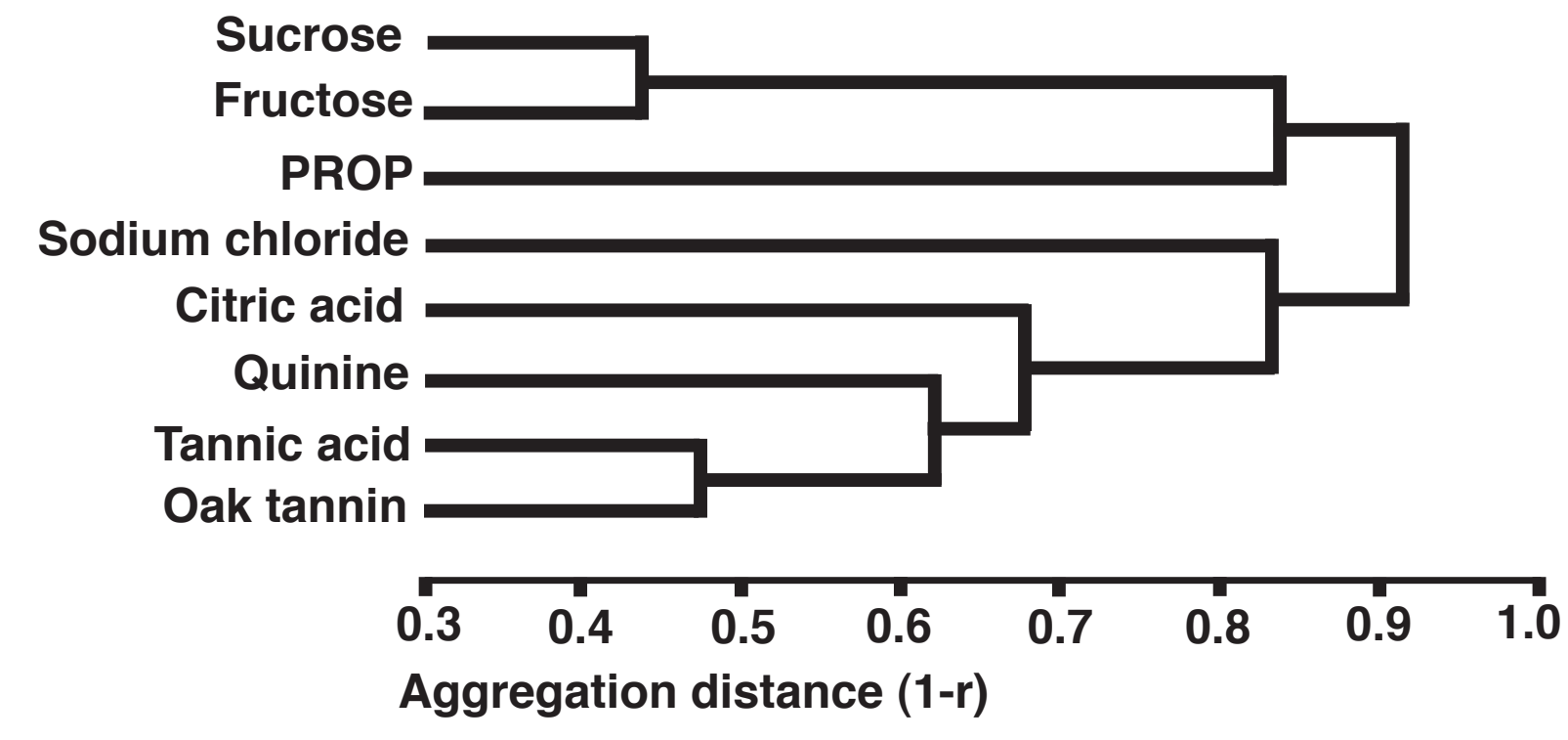

FIGURE 3 - Cluster tree of taste thresholds (as log; average linkage method, using Pearson correlation coefficients to determine aggregation distances) for various subtances tested on 123 adults in Tunis.

Food preferences and attitudes: Food items were actually tested from a list and not tasted; however, it noticeable that supra-threshold mean hedonic values for solutions of salt and sugars do correlate with preference self report (respectively $\mathrm{r}=0.30 ; \mathrm{p}=0.001$, and $\mathrm{r}=0.44 ; \mathrm{p}=0.0001$ ).

As shown in Figure 5, most of the food items are perceived positively. This was an expected result, since the list concerns the most representative food items of the Tunisian diet. Most of the items negatively rated were either never eaten (such as the grapefruit), or had a peculiar cultural status (such as alcoholic beverage in a Muslim society) for several of the tested subjects. The highest ranked items, such as olive oil, fish and oranges, are the most endogenous. Age is positively correlated to the mean reported food preferences $(r=0.26 ; p=0.004)$. The eldest gave higher rating for 10 items, such as rancid butter $(\mathrm{r}=0.30 ; \mathrm{p}=0.002)$, olive oil $(\mathrm{r}=0.28 ; \mathrm{p}=0.002)$, and coffee $(\mathrm{r}=0.20$; $\mathrm{p}=0.04)$, out of the list of 43 items. Conversely one food item, soda drink was not rated as high by the eldest than by

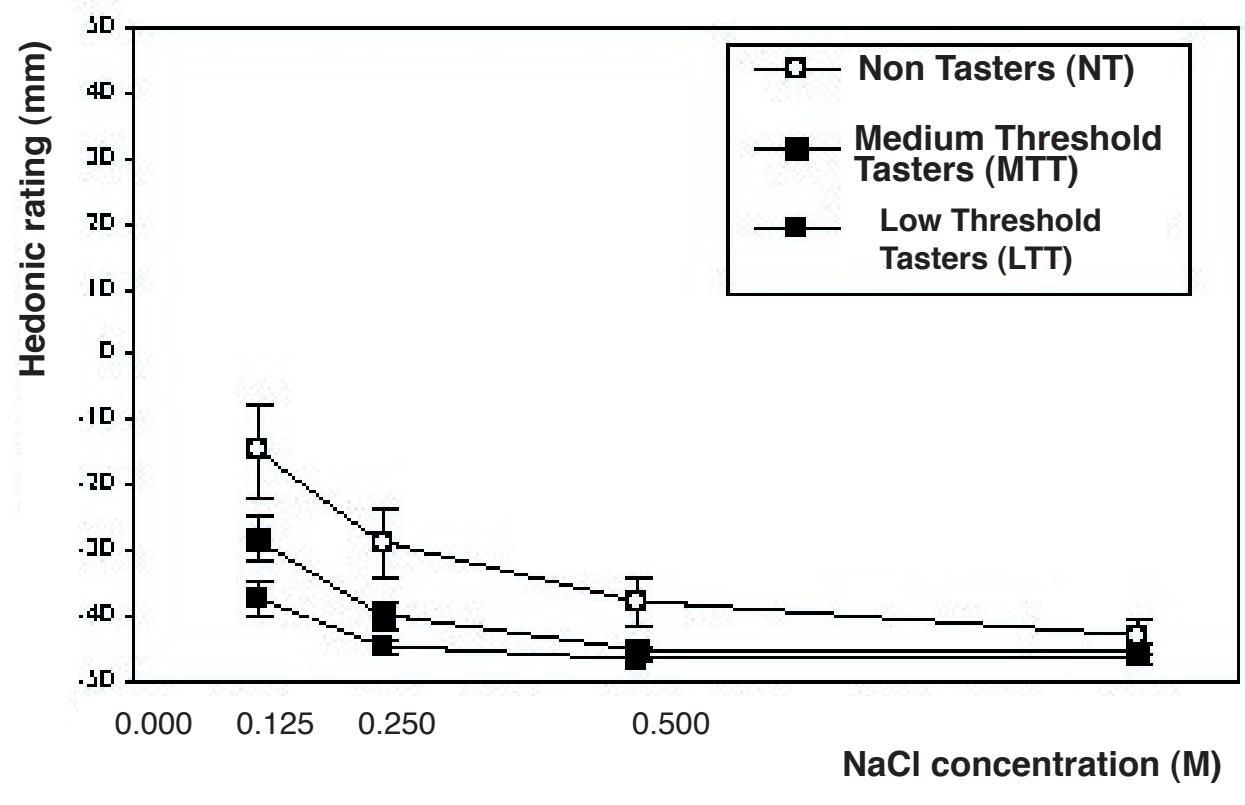

FIGURE $4-$ Hedonic ratic of sodium chloride solutions at increasing concentrations, according to PROP sensitivity categories. 


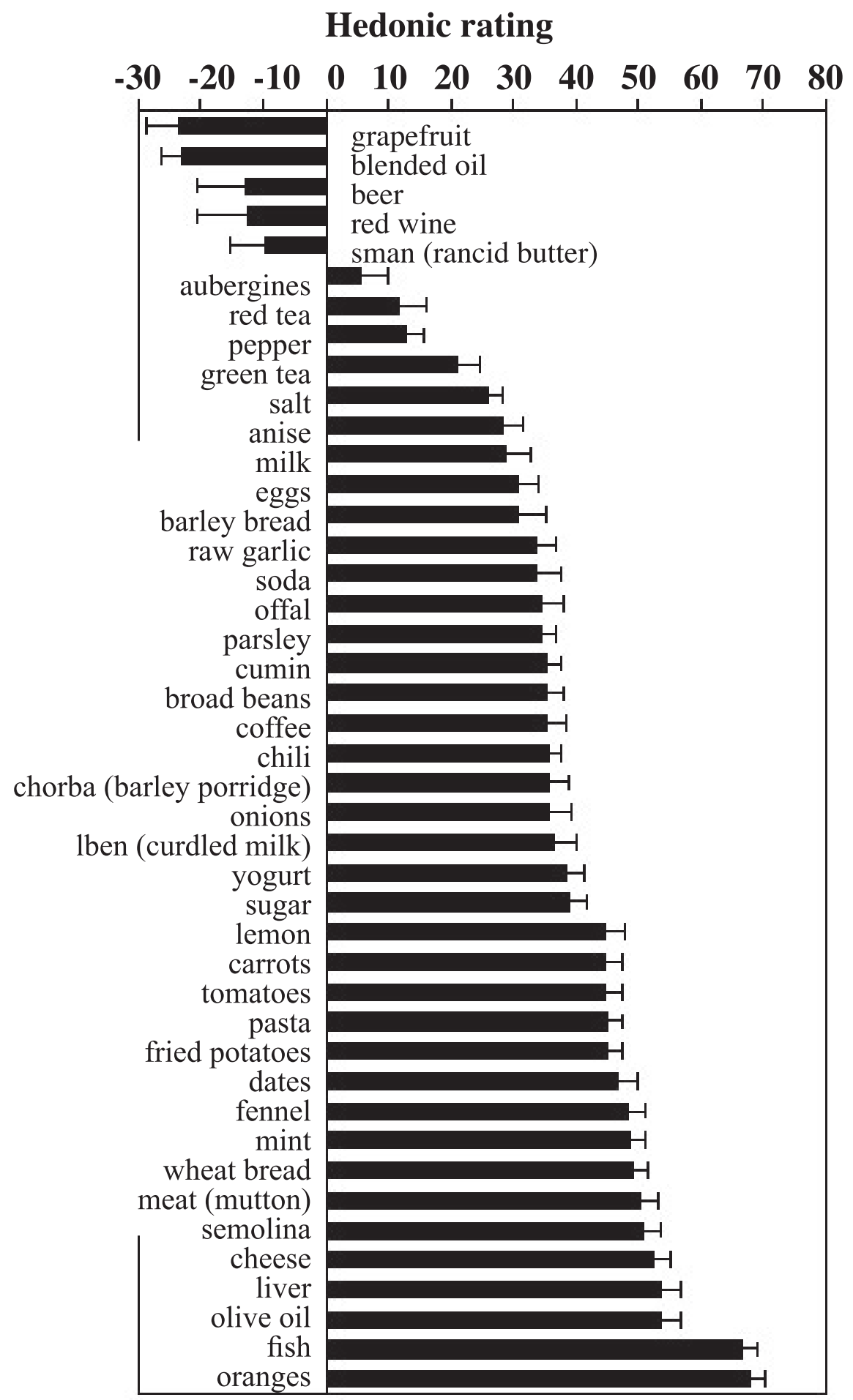

FiguRE 5 - Hedonic rating of the food items included in the Tunisian food list

the youngest subjects. Sex differences were noted for 7 items out of 43 , including alcoholic beverages and onions, preferred by men. In addition, men actually know or consume a larger number of food items than women $(\mathrm{t}=2.28$; $\mathrm{p}=0.002$ ). Many of the preferred foods are also considered as the healthiest, by both sexes. Conversely, food cost, prestige and festive use are diversely matched with food preference ratings.

\section{PROP status and food preferences}

Taking into account the three categories of PROP sensitivity, we found that low threshold tasters (LTT) tend to rate preference for foods higher than the other subjects (MTT + NT). As shown in Table I, significant differences are found for 14 items out of 43 , and positive for 12 , as well as for the mean of preference ratings. If we except green 
tea, which is perceived as less pleasant by PROP tasters as a group (LTT + MTT), none of the food item known as containing bitter phytochemicals of interest for health (Drewnowski, 2000), such as grapefruit, coffee, red wine, are not differentially appreciated by the various categories of PROP tasters.

\begin{tabular}{lccc} 
& $(\mathrm{LTT}+\mathrm{MTT}) \mathrm{vs}$ NT & LTT vs (MTT+NT) \\
& $\mathrm{t}$ & $\mathrm{t}$ & \\
\hline Barley bread & -0.60 & 2.17 & $* *$ \\
Chorba & $-2.82 * *$ & -0.26 & \\
Aubergine & -1.28 & 2.95 & $* *$ \\
Oranges & 0.89 & 2.01 & $*$ \\
Liver & 1.20 & $2.90 * *$ \\
Offal & 0.29 & $2.01 *$ \\
Eggs & 0.58 & $3.22 * *$ \\
Milk & $2.47 *$ & -1.13 \\
Lben (curdled milk) & -0.74 & $2.24 *$ \\
Sman (rancid butter) & -1.26 & $2.23 *$ \\
Anise & $2.47 *$ & $2.02 *$ \\
Chili & -1.28 & $2.33 *$ \\
Mint & 0.20 & $2.20 *$ \\
Green Tea & $-2.40 *$ & -0.92 & \\
& & 3.17 & $* *$ \\
Mean of the preference ratings & -0.25 & $-2.05 *$ \\
Nb. of food items actually consumed & -1.59 & -0.01 & \\
\% food items perceived as unpleasant & 1.16 & & \\
\hline
\end{tabular}

${ }^{*} p<0.05 ;{ }^{* *} p<0.001$

TABLE 1. - $t$ values of the $t$ test of the differences between groups of PROP tasters, for preference ratings in tunisian food items. For all other foods in the list of 43 items, no significant difference is observed.

\begin{tabular}{|c|c|c|c|}
\hline Sex & Age & $\begin{array}{l}\text { PROP sensitivity } \\
\text { LTT vs (MTT+NT) }\end{array}$ & $\begin{array}{l}\text { Health value } \\
\text { of food }\end{array}$ \\
\hline
\end{tabular}

\section{Cost Ritual/ \\ Prestige}

TABLE 2. - Beta coefficients and \% explained variation from the multiple regression analysis of biological and attitudinal factors on the mean reported preference in the food list.

The percentage of food items perceived as unpleasant does not differ significantly between the groups of tasters. Interestingly, LTT actually consume a significantly lower number of food items. In contrast, no specificity in food preference ratings was found among PROP tasters as a group (LTT + MTT), compared with NT. 
Table II shows the result of a multiple regression analysis of various predictive factors on the mean reported food preferences. Attitudinal variables were reduced into health, cost, and ritual/prestige factor-based variables, using principal components factor analysis. Significant positive beta coefficients $(\mathrm{p}<0.05)$ are found for age, mean health value of food, and PROP taste sensitivity (LTT vs. MTT + NT). Thus, the PROP taste factor is linked to a higher mean food preference rating, independently from the other biological and attitudinal variables included into the analysis.

\section{DisCUSSION}

From a random sample of adult inhabitants of Tunis, the measurement of 6- $n$-propylthiouracil (PROP) taste perception showed that, separating the subjects according to the underlying statistical distribution of taste recognition thresholds brings out the specificity of the subjects with a low taste threshold (LTT). This specificity concerns: (i) a trend towards higher taste sensitivity for the other substances tested, (ii) higher perceived unpleasantness of $\mathrm{NaCl}$ (a stimuli generally rated as aversive), (iii) a trend to rate food preferences (from a checklist of food items generally rated as pleasant) higher than other groups, irrespective from sex, age, and cultural factors, (iv) a trend towards less food items actually used or known in the checklist.

(i) The trend towards higher taste sensitivity for substances such as sugars, quinine, tannins, etc., observed in LTT as compared to the other subjects, suggests the very existence of a genetic system with gene(s) coding for global taste sensitivity, besides the gene coding for PROP sensitivity, as proposed by Olson et al. (1989). However, the weak aggregation of PROP sensitivity with that of other substances and especially quinine, shown in Figure 3 , argues in favour of the interpretation of Delwiche et al. (2001), who consider various bitter substances as stimulating partly similar taste receptors. In a recent paper (Hladik et al., 2002), we also described the relationships between taste thresholds in a large sample of human adults for various tastants, showing that the observed structure of the cluster tree is a robust model, including the strong relationships between quinine and tannins, a likely inheritance of primate taste receptors evolution.

(ii) The clear-cut relationship between PROP sensitivity and hedonic perception of $\mathrm{NaCl}$ described in this paper has never been found in earlier studies (Drewnowski et al., 1997a). Such a discrepancy could be explained by a different methodology: in our study, the salty solutions were presented in ascending concentration order, a protocol reducing variability as compared to randomised order. The results yielded by such different methods have been discussed by Garner (1953), showing that the subjects tend to note according to the previous taste signal. This second order effect was not cancelled in our study, but the method appear as facilitating the expression of the underlying group effect.

(iii) Higher rating in individual food preferences found in the LTT group is another novel aspect of the specificity of PROP sensitivity. Comparing PROP tasters as a group (LTT+MTT) with non-tasters, no difference is found in both preferences and number of dislikes. Such results contrast with those of early studies reporting that individuals exhibiting a high sensitivity to PROP tend to show more dislikes for common foods than do non-tasters (Fisher et al., 1961). However, some later studies linking PTC and PROP sensitivity to food preferences and use, especially for vegetable, are much less conclusive (Niewind et al., 1988; Jersza-Latta et al., 1990), providing data comparable to ours.

Further methodological differences might, again, explain the various results. In previous studies, the most widely used scale for assessing food likes/dislikes was the nine-point hedonic scale (Peryam \& Pilgrim, 1957), which results in a reduced ability to differentiate among highly liked or disliked foods. The Labelled Affective Magnitude (LAM) scale used in our study has an equal reliability and sensitivity to the nine-point scale and provide a greater discrimination among the highly liked foods (Schutz \& Cardello, 2001). The use of the LAM scale allowed to shed light on higher rankings of foods by the LTT.

The finding that mean preference ratings of LTT are independent from sex, age and attitudinal/cultural factors, highlights the importance of the congenital factors in the adult food choices. 
(iv) Most of the items unknown or unused, especially by LTT were associated with low acceptance ratings. The trend towards less food items actually used or known in the checklist among LTT, together with higher food preference ratings, suggest that LTT could have difficulties to overcome an inherent neophobia. LTT prefer the most familiar foods, although those are, for them, in limited number, but they could be more reluctant with unfamiliar items. Further investigation should include more unfamiliar or presumably disliked food items than our consensual food list.

Before our attempt to use the underlying distribution of the PROP taster in three groups, and our finding of the particular responses of the LTT, Bartoshuk (1993) distinguished a group of "super-tasters" among the PROP tasters, from detection thresholds and the mean ratio of intensity rating of supra-threshold PROP solutions relative to sodium chloride solutions. This group of super-tasters exhibit a reduced acceptability for bitter substances and several foods containing plant-based toxins (i.e. grapefruit juice, green tea, etc.; Akella et al., 1997; Drewnowski et al., 1997b). Interestingly, this trend could be related to the selective pressure exerted on the taste genes of our remote primate ancestors, resulting in the avoidance of potentially toxic foods. However, the background of primate taste evolution (Hladik et al., 2002) does not show any relationship between the ability to taste bitter or astringent substances such as quinine and tannins, evolved in plants to deter consumers, and sensitivity to the artificial chemical, PROP.

What could account for the food behaviour of the LTT in terms of taste genetics, considering that PROP recognition thresholds may only partly reflect the result of selective pressures on primate taste system? Gene coding for taste is obviously much more complex that was initially supposed. In PROP sensitivity, as suggested by Olson et al. (1989), at least two groups are involved: one with a specific sensitivity to PROP (supertasters, eventually including a part of the LTT), and another group with a high sensitivity to a wide range of sensory stimuli, the LTT. In this last group, the sensory genetically mediated factor influences food acceptance irrespective of socio-cultural factors, and can be used, to some extent, as a predictor of food choices.

\section{Acknowledgments}

The authors are indebted to the staff of the Nutrition Institute of Tunis, for helping them in this study, and to the volunteers, who kindly paticipated in the tests.

\section{REFERENCES}

Ake山a, G.D., Henderson, S.A. \& Drewnowski, A. (1997). Sensory accep tance of Jap Anese green tea And soy p RodUCTS IS UNKED TO GENETIC SENSITIVITYTo 6- $n$-propylthiouracil. Nutrition and Cancer, 29: 146-151.

Bartoshuk, L.M. (1993). The biological basis of food perception and acceptance. Food Quality and Preference, 4: 21-32.

Birch, L.P., Zimmerman, S.I. \& Hind, H. (1980). The influence of social-affective context on the formation of children's food preferences. Child Development, 51: 856-861.

Cornsweet, T.N. (1962). The staircase-method in psychophysics. American Journal of Psychology, 75: 485-491.

Delwiche J.F., Buletic Z., Breslin P.A.S. (2001). Covariation in individuals' sensitivities to bitter compounds: evidence supporting multiple receptors/transduction mechanisms, Perception and Psychophysics, 63: 761-776.

Drewnowski, A. (2000). Bitter taste, phytonutrients, and the consumer: a review. American Journal of Clinical Nutrition, 72: 1424-1435.

Drewnowski, A., Henderson, S.A., Shore, A.B. \& Barrat-Fornell, A. (1997a). Nontasters, tasters, and supertasters of 6-n-propylthiouracil (PROP) and hedonic response to sweet. Physiology \& Behavior, 62: 649-655.

Drewnowski, A., Henderson, S.A., \& Shore, A.B. (1997b). Taste responses to naringin, a flavonoid, and the acceptance of grapefruit juice are related to genetic sensitivity to 6- $n$-propylthiouracil. American Journal of Clinical Nutrition, 66: 391-397.

Fisher, R., Griffin, F., England, S. \& Garn, S.M. (1961). Taste thresholds and food dislikes. Nature, 191: 1328.

Fox, A.L. (1932). The relatioship between chemical constitution and taste. Proceedings of the National Academy of Sciences (Washington), 18: 115-120.

Garner, W.R. (1953). An informational analysis of absolute judgments of loudness. Journal of Experimental Psychology, 46: 373-380. 
Hladik, C.M. \& Pasquet, P. (1999). Évolution des comportements alimentaires : adaptations morphologiques et sensorielles. Bulletins et Mémoires de la Société d'Anthropologie de Paris, n.s.11: 307-332.

Hladik, C.M., Pasquet, P. \& Simmen, B. (2002) - New perspectives on taste and primate evolution: the dichotomy in gustatory coding for perception of beneficent vs. noxious substances as supported by correlations among human thresholds. American Journal of Physical Anthropology, 117: 342-348

Hubert, A. (1984). Le pain et l'olive, aspects de l'alimentation en Tunisie. Editions du CNRS, Paris

Jersza-Latta, M., Krondl, M. \& Coleman, P. (1990). Use and perceived attributes of cruciferous vegetables in terms of genetically-mediated teste sensitivity. Appetite, 15: 127-134.

Maclean, C.J., Morton, N.F., Elston, R.C. \& Yee, S. (1976). Skewness in comingled distributions. Biometrics, 32 : 695-699.

Niewind, A., Krondl, M. \& Shrott, M. (1988). Genetic influence on the selection of Brassica vegetables by elderly individuals. Nutrition Research, 8: 13-20.

Olson, J.M., Boehnke, M., Neiswanger, K. Roche, A.F. \& Siervogel, R.M. (1989). Alternative genetic models for the inheritance of the phenylthiocarbamide (PTC) taste deficiency. Genetic Epidemiology, 6: 423-434.

Pasquet, P. (1994). Existe-t-il une héritabilité génétique de la perception gustative, des préférences alimentaires et de la prise alimentaire? In Atelier du goût, Compte-Rendu 1994, pp. 95-108. Paris, ANVIE, Maison des Sciences de l'Homme.

Peryam, R.D. \& Pilgrim, J.F. (1957). Hedonic scale method for measuring food preferences. Food Technology, 11: 9-14.

Reed, D.R., Bartoshuk, L.M., Duffy, V. \& Price, R.A. (1995). Propylthiouracil tasting: determination of underlying threshold distributions using maximum likelihood. Chemical Senses, 20: 529-533.

Reddy, F.E, Bartoshuk, L.M., Miller, I.J., Duffy, V.B. \& Yanagisawa, K. (1993). Relationship

among papillae, taste pores and 6-N-propylthiouracil (PROP) suprathreshold taste sensitivity. Chemical Senses, 18: 619-619.

Rozin, P. (1990). Acquisition of stable food preferences. Nutrition Reviews, 48: 106-113.

Schutz, H.G. \& Cardello, A.V. (2001). A labeled affective magnitude (LAM) scale for assessing food liking/disliking. Journal of Sensory Studies, 16: 117-159.

Smagghe, K. \& Louis-Sylvestre J. (1998). Influence of PROP-sensitivity on taste perceptions and hedonics in French women. A study performed without retronasal olfaction. Appetite, 30: 325-339.

Steiner, J.E., Glaser, D., Hawilo, M.E. \& Berridge, K.C. (2001). Comparative expression of hedonic impact; affective reactions to taste by human infants and other primates. Neuroscience and Behavioral Reviews 25: 53-74. 